\title{
Repair of Oronasal Fistulae by Interposition of Multilayered Amniotic Membrane Allograft
}

Nils H. Rohleder, M.D., D.D.S

Denys J. Loeffelbein, M.D., D.D.S., Ph.D.

Wolfgang Feistl, M.D., D.D.S. Matthias Eddicks, D.V.M.

Klaus-D. Wolff, M.D., D.D.S., Ph.D. Aakshay Gulati, F.D.S.R.C.S., M.R.C.S., F.R.C.S.

Lars Steinstraesser, M.D.,

Ph.D.

Marco R. Kesting, M.D.,

D.D.S., Ph.D.

Munich, Oberschleissheim and Bochum, Germany; Linz, Austria; and Southampton, Hampshire, United Kingdom

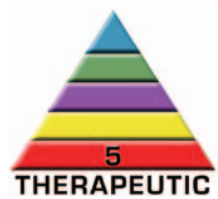

Background: Oronasal fistulas are a frequent complication after cleft palate surgery. Numerous repair methods have been described, but wound-healing problems occur often. The authors investigated, for the first time, the suitability of multilayered amniotic membrane allograft for fistula repair in a laboratory experiment (part A), a swine model (part B), and an initial patient series (part C).

Methods: In part A, one-, two-, and four-layer porcine and human amniotic membranes $(n=20$ each) were fixed in a digital towing device and the force needed for rupture was determined. In part B, iatrogenic oronasal fistulas in 18 piglets were repaired with amniotic membrane allograft, autofetal amniotic membrane, or small intestinal submucosa $(n=6$ each). Healing was evaluated by probing and visual inflammation control (no/moderate/ strong) on postoperative days $3,7,10$, and 76 . Histological analysis was performed to visualize tissue architecture. In part C, four patients (two women and two men, ages 21 to 51 years) were treated with multilayered amniotic membrane allograft.

Results: In part A, forces needed for amniotic membrane rupture increased with additional layers $(p<0.001)$. Human amniotic membrane was stronger than porcine membrane $(p<0.001)$. In part $\mathrm{B}$, fistula closure succeeded in all animals treated with amniotic membrane with less inflammation than in the small intestinal submucosa group. One fistula remained persistent in the small intestinal submucosa group. In part C, all fistulas healed completely without inflammation.

Conclusions: Amniotic membrane is an easily available biomaterial and can be used successfully for oronasal fistula repair. The multilayer technique and protective plates should be utilized to prevent membrane ruptures. (Plast. Reconstr. Surg. 132: 172, 2013.)

CLINICAL QUESTION/LEVEL OF EVIDENCE: Therapeutic, V. ronasal fistula development is a complication after cleft palate surgery, with an incidence of 3.9 to 4.0 percent. ${ }^{1,2}$ Further causes are trauma, tumors, infections, or nasally administered drugs, such as cocaine. ${ }^{3-6}$ Patient

From the Department of Oral and Maxillofacial Surgery, Technische Universität München; Department of Oral and Maxillofacial Surgery, General Hospital of Linz; Clinic for Swine, Faculty of Veterinary Medicine, Ludwig-Maximilians-University of Munich; Department of Oral and Maxillofacial Surgery, Southampton University Hospital NHS Foundation Trust; and Department of Plastic Surgery, Burn Unit, BG University Hospital Bergmannsheil, Ruhr University Bochum.

The first two authors contributed equally to this study. Received for publication December 17, 2012; accepted January $28,2013$.

Copyright $(2013$ by the American Society of Plastic Surgeons

DOI: 10.1097/PRS.0b013e3182910b50 discomfort is caused by saliva and food inflow into the nasal cavity, velopharyngeal insufficiency, and hypernasality of speech. ${ }^{7-9}$

Numerous techniques for oronasal fistula repair have been described, including tongue flaps, ${ }^{10-14}$ mastoid fascia grafts, ${ }^{15}$ auricular cartilage transplantation, ${ }^{16}$ bone grafts, ${ }^{17}$ distraction osteogenesis, ${ }^{18}$ and free flap transfer. ${ }^{19,20}$ However, no standard techniques have been presented, and up to 65 percent of oronasal fistulas are reported to reappear. ${ }^{21}$ This might be attributable to local tissue conditions, with scarring and restricted motility making tension-free surgical intervention difficult. Current research is aimed at developing tension-reductive techniques.

Disclosure: The authors have no financial interest to declare in relation to the content of this article. 
In 2006, Kirschner et al. ${ }^{21}$ introduced the use of human acellular dermal matrices for oronasal fistula repair. The authors observed the ingrowth of fibrovascular tissue and complete fistula closure in nine patients.

Another biomaterial suitable for oronasal fistula repair might be amniotic membrane, which is easily available and reasonably priced with regard to its harvesting and processing to a medical product (e.g., testing for viral infections of the donors and cryoconservation). Amniotic membrane is the inner layer of the fetal membranes and has been investigated as an alternative biomaterial for various purposes in reconstructive surgery since its initial description as a material suitable for transplantation in $1910 .{ }^{22}$ It offered new perspectives, for example, in the treatment of burn wounds. ${ }^{23}$ Interest in human amniotic membrane diminished as a consequence of emerging awareness of human immunodeficiency virus and the fear of virus transmission in the 1980s. The end of the 1990s saw the establishment of new methods for the processing and long-term storage (such as cryoconservation) of human amniotic membrane, and its use in reconstructive surgery became popular again. ${ }^{24}$ However, the material is thin and lacerable, and its use in the oral cavity is challenging (e.g., because of forces caused by the tongue and food particles).

We recently investigated the suitability of xenogenous human amniotic membrane for the repair of oronasal fistula in a porcine model and obtained promising results. ${ }^{25}$ The aim of the present study was to expand our knowledge of this material as follows: (1) by evaluating the adequacy of the tensile strength of human and porcine amniotic membrane when arranged in the multilayer technique; (2) by evaluating amniotic membrane allograft for oronasal fistula repair in a porcine model and investigating possible advantages of autofetal amniotic membrane (i.e., the "autologous" amniotic membrane of a fetus harvested at its birth) (as a control, a resorbable, extracellular matrix scaffold material, termed "small intestinal submucosa," was also evaluated); and (3) by transference to clinical practice in an initial small series of patients.

\section{METHODS}

\section{Porcine and Human Amniotic Membrane}

Experiments on animals were conducted in accordance with German governmental regulations for animal welfare and with approval of the District Council (no. 55.2-1-54-2531-183-09; April 26, 2010). Porcine amniotic membrane was

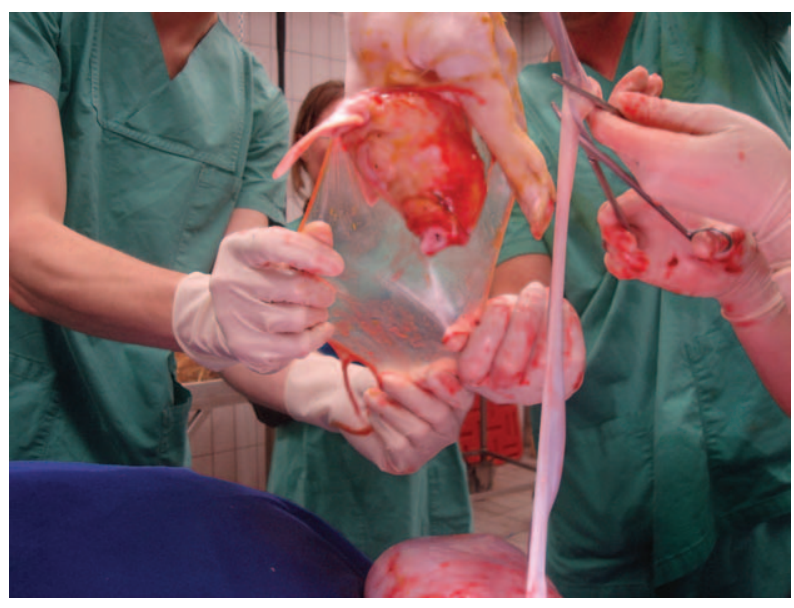

Fig. 1. Harvest of porcine amniotic membrane. Careful separation of the membrane from the piglet is carried out under sterile conditions.

harvested during porcine cesarean delivery carried out under general anesthesia, in the Clinic for Swine of Ludwig-Maximilians-University Munich. The membranes were separated from the piglets on their removal from the uterus under sterile conditions (Fig. 1), transferred to a sterile laminar flow hood (Herasafe; Kendro, Langenselbold, Germany), cut into $5 \times 5$-cm pieces, and applied to nitrocellulose sheets (Raucocel; Lohmann-Rauscher, Rengsdorf, Germany). Each sheet was stored in a 50-ml sterile tube (Falcon; BD Biosciences, San Jose, Calif.) containing medium for cryoconservation consisting of $250 \mathrm{ml}$ of glycerin, $250 \mathrm{ml}$ of Dulbecco's Modified Eagle Medium (JR Scientific, Woodland, Calif.), $5 \mathrm{ml}$ of penicillin/streptomycin solution, and $5 \mathrm{ml}$ of amphotericin B solution (Merck, Darmstadt, Germany). The tubes were stored at $-80^{\circ} \mathrm{C}$ and thawed for 30 minutes before usage in a $28^{\circ} \mathrm{C}$ water bath.

The study protocols for the use of human amniotic membrane in human patients were approved by the District Council and the local ethics committee (no. 3071/10; February 1, 2011). Operations were carried out in accordance with the Declaration of Helsinki. All patients gave written informed consent. Human amniotic membrane allografts were fabricated in cooperation with the Red Cross Transfusion Service of Upper Austria, Linz, Austria, as a certified medical product at a size of $4 \times 4 \mathrm{~cm}$. This size was determined in unpublished internal studies to ideally meet the requirements of reconstructive surgery in the oral cavity. Agglutination tests were performed for human immunodeficiency virus (anti-HIV), hepatitis C (anti-HCV), hepatitis B (anti-HBc, HB-Ag), and 
cytomegalovirus (anti-CMV-IgG/-IgM); Treponema pallidum hemagglutination and enzyme-linked immunosorbent assay tests were performed for syphilis; and polymerase chain reaction tests were performed for human immunodeficiency virus, hepatitis $\mathrm{C}$ virus, and human papilloma virus.

\section{Part A: Laboratory Experiment}

Uniaxial forces needed for the rupture of onelayer, two-layer, and four-layer human and porcine amniotic membranes were evaluated by fixing the membranes on a standardized square field $(3 \times 3 \mathrm{~cm})$ in a digitally controlled towing device (model 100kN; Zwick, Ulm, Germany) (Fig. 2). Computer software determined the maximal force applied until rupture. The first test series consisted of 20 repeats for each one-layer, twolayer, and four-layer porcine membrane. In the second series, the experiment was repeated with human amniotic membrane.

Statistical Analysis of Laboratory Experiment IBM SPSS 19.0 software (SPSS, Inc., Chicago, Ill.) was used. Differences with respect to the forces needed for membrane rupture were analyzed by using the Mann-Whitney $U$ test. All $p$ values are given as two-tailed with a local significance level of 5 percent.

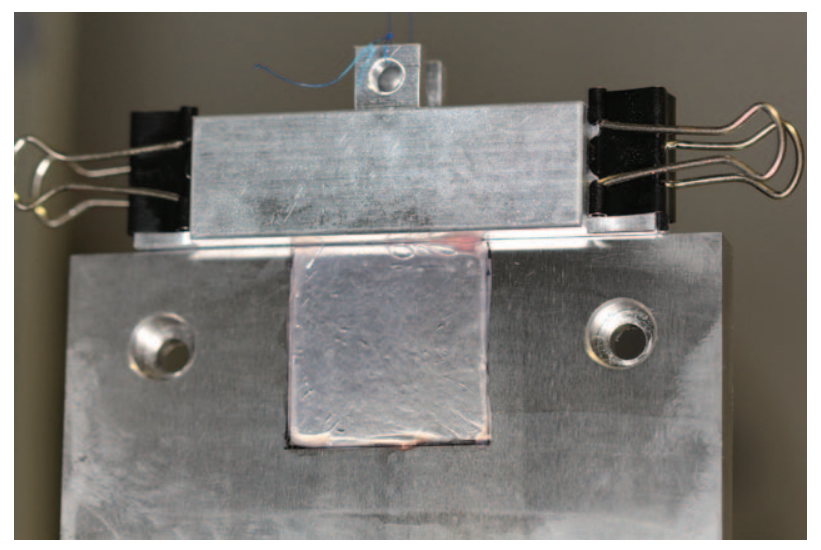

Fig. 2. Uniaxial forces needed for rupture of one-, two-, and fourlayer human and porcine amniotic membrane were evaluated by fixing the membranes on a standardized test field with a width of $3 \mathrm{~cm}$ in a digitally controlled towing device (model $100 \mathrm{kN}$; Zwick, Ulm, Germany). The lower part of the image shows an example of a single-layer amniotic membrane adapted to the immobile metal plate of the machine. Membranes were subsequently fixed to this plate by the addition of a second metal plate that was tightened against the first plate. The clamp in the upper half of the image was connected to the mobile part of the towing machine and pulled the membrane upward once the experiment was initiated. Forces needed until rupture were digitally recorded by computer software.

\section{Part B: Animal Experiment}

\section{Animals and Defect Preparation}

Twenty-four 8-week-old piglets ("Schwaebisch Haellische" × "Pietrain" hybrids, 22 to $32 \mathrm{~kg}$; kept according to the international principles of laboratory animal care) were used. All were born by cesarean delivery from the same dam in the Clinic for Swine of Ludwig-MaximiliansUniversity, Munich. Their amniotic membrane was harvested and stored as autofetal amniotic membrane. Amniotic membrane allografts were collected from another dam. Water and food were given freely. Oronasal fistulas were produced under general anesthesia. To induce anesthesia, $10 \mathrm{mg} / \mathrm{kg}$ body weight ketamine (Ursotamin; Serum-Werk, Bernburg, Germany) and $2 \mathrm{mg}$ / $\mathrm{kg}$ azaperone (Stresnil; Janssen-Cilag, Neuss, Germany) were administered intramuscularly. Animals were intubated, and anesthesia was maintained with 1 to 2 percent isoflurane (Isoba; Intervet, Unterschleissheim, Germany) and 3 to 5 liters/minute oxygen. Piglets were placed on their back. Silicon dental casts of the palates were taken before fistulas were made in order to manufacture individual protection plates for coverage of the defects after repair. The plates were manufactured in the Laboratory of the Technische Universität München and consisted of polymethylmethacrylate. In each piglet, a full-thickness defect in the mid-hard palate of $\sim 15 \mathrm{~mm}$ in diameter was produced with a biopsy punch and subsequent watercooled osteotomy of the hard palate (Fig. 3). The nasal septum and edges of the bony palate were rongeured beyond the mucosal edges to allow for re-epithelialization at the periphery of the defects. Fistulas matured for 2 weeks. After this time, all fistulas were persistent and re-epithelialized at their margins.

\section{Fistula Repair}

Six animals were randomly selected to have their fistulas repaired with allografts of fivelayer amniotic membrane, six were treated with five-layer autofetal amniotic membrane, and six received small intestinal submucosa (Cook Group Inc., Bloomington, Ind.). Multilayered amniotic membrane was prepared as previously described ${ }^{26}$ Grafts were interposed between the oral and nasal mucosa traversing the palatal fistulas and sutured with polyglactin (Vicryl 4-0; Ethicon, Norderstedt, Germany) to the lateral edges of the mucoperiosteal bilateral flap created according to the von Langenbeck technique (Fig. 4). Mucosal edges of the fistulas were 


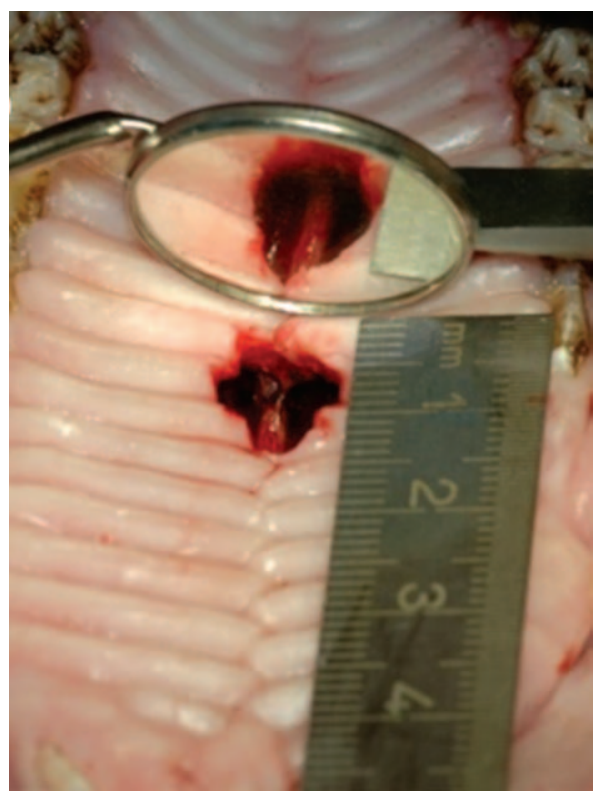

Fig. 3. Creation of iatrogenic oronasal fistula in the porcine palate. The animal had previously been intubated and placed on its back, with its tongue fixed to the lower jaw. The nasal septum is visible in the middle of the fistula. The marginal bone was removed in an undermining manner to allow epithelial growth over the defect margins from both the palatine and the nasal side of the fistula. In this manner, so-called critical-size defects were created, which showed no tendency for spontaneous healing. The defects were allowed to mature for 2 weeks and were subsequently repaired with multilayered amniotic membrane allograft, autofetal amniotic membrane, or small intestinal submucosa.

not closed. The prefabricated protective plates were placed onto the palates and fixed with two 13-mm screws in order to protect the transplants from mechanical damage (Fig. 5). Wounds were monitored by photodocumentation on postoperative days $3,7,10$, and 76 under short sedation. They were cleaned of blood clots and food pellets after removal of the palatal protective plates, which were refixed until they were ultimately removed on day 10 . Wound monitoring involved the assessment of local inflammation by visual grading of the degree of redness around the fistula $(0=$ none; $1=$ moderate $; 2=$ pronounced $)$ in the photographs, which were scored independently by two blinded investigators. On day 76, the pigs were euthanized by sedation and intravenous administration of $45 \mathrm{mg} / \mathrm{kg}$ pentobarbital (Release; WdT, Garbsen, Germany). A final evaluation of the palates for remaining fistulas was performed with a probe, and the palates were removed and stored in formalin (3.7 percent) for microscopic analyses.

\section{Histological Analysis}

Samples were embedded in paraffin, and 4- $\mu$ m-thick sections were cut with a microtome (Hyrax M 55; Zeiss, Jena, Germany). Paraffin was extracted, and sections were stained with hematoxylin and eosin (Bio Optica, Milan, Italy) and analyzed with a microscope (AxioCam HRC; Zeiss). A closed oral and nasal epithelium with no fistula remnants and no signs of inflammation was regarded as the preferred result. Microscopic evaluation of the former defect region on day 76 was carried out in order to confirm the clinical results with respect to closure of the oral epithelium and to investigate the closure of the nasal defects and the presence of fistula tract remnants on either side of the nasal septum. Accordingly, two regions were evaluated in each animal,
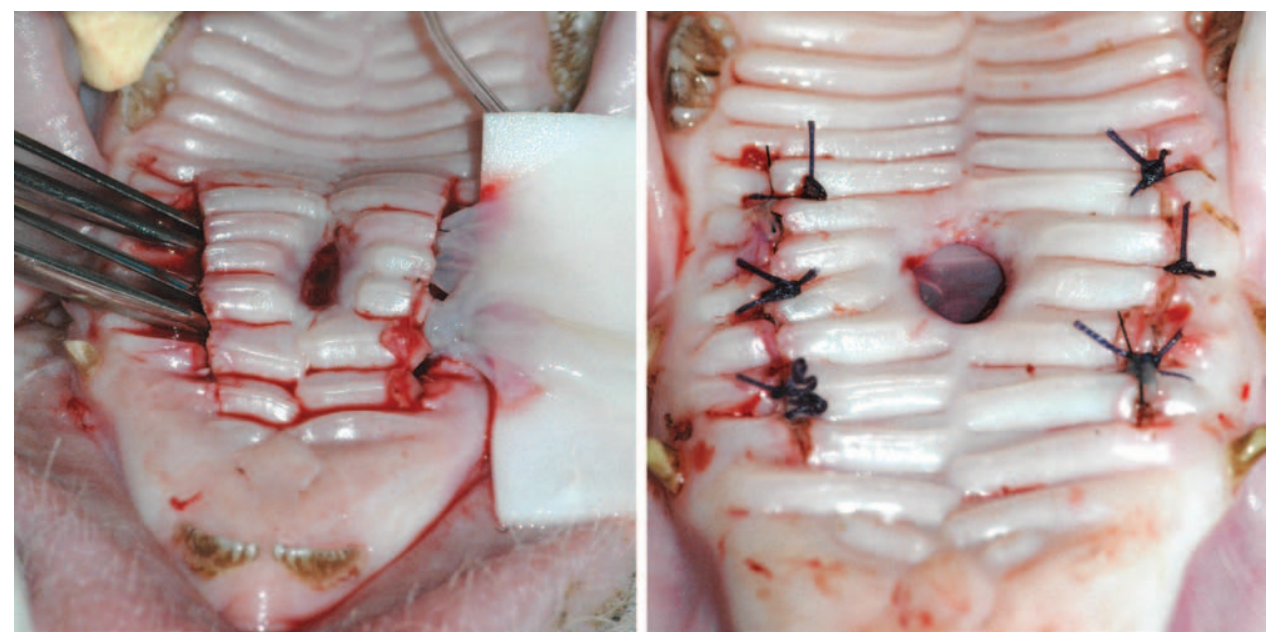

Fig. 4. Repair of iatrogenic oronasal fistula in a palate of a piglet using the von Langenbeck technique. (Left) Multilayered amniotic membrane is gently pulled through the incisions. (Right) The defect is covered with the transplant. 


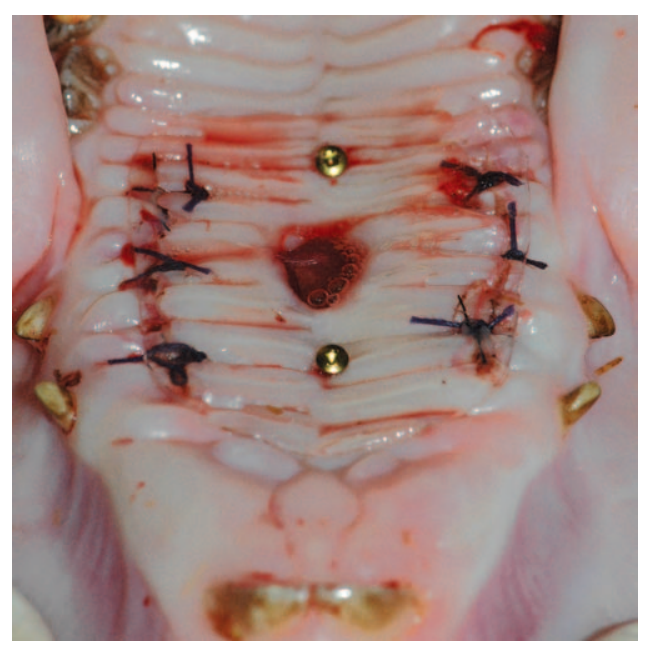

Fig. 5. Fixed polymethylmethacrylate protective plate in the palate of a piglet to protect the transplants from mechanical damage caused by food or hay.

resulting in a total of 12 areas of interest in each group. All stainings were produced in duplicate and scored independently by two blinded investigators.

\section{Part C: Clinical Trial}

Four patients (two women and two men, ages 21 to 51 years) diagnosed with oronasal fistula of the hard palate were treated with multilayered amniotic membrane allografts (three in Munich, one in Linz). After defect preparation (removal of epithelium covering the fistula margins), amniotic membrane was fixed to the marginal oral epithelium (the fistula edges were freshened and the amniotic membrane was sewn to the nasal epithelium and subsequently to the oral mucosa) (Fig. 6, above). In none of the four patients could the defect be closed primarily or by local flaps. The von Langenbeck technique was not used in the patients in an attempt to reduce local tissue trauma. Protective plates were inserted for transplant protection in each patient (Fig. 6, center). The plates were produced as follows: dental models of the maxilla were produced by obtaining impressions in alginate (Palgat; 3M, Neuss, Germany) and filling them with gypsum. Fistulas were closed in the models with dental wax in order to simulate the postoperative situation. Polymethylmethacrylate plates (Biocryl; Scheu, Iserlohn, Germany) were heated and suctioned over the models in a vacuum device (Twinstar; Scheu), as recommended by the manufacturer. After the plates cooled down, their edges were shortened in order to avoid gingival trauma.
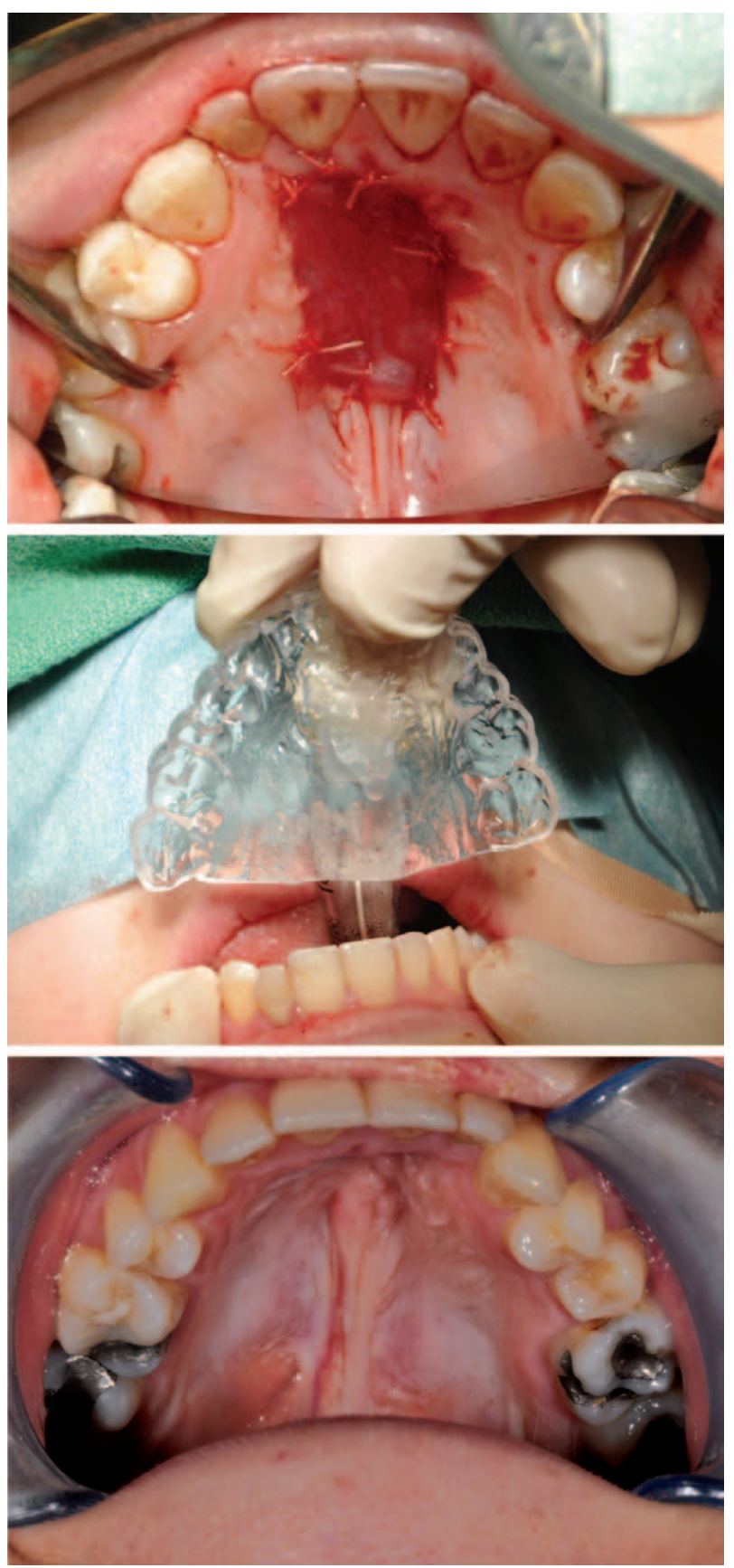

Fig. 6. Clinical use of amniotic membrane allograft for closure of a large oronasal fistula in a patient with a previously operated cleft palate. (Above) Amniotic membrane covering defect, fixed with sutures. (Center) Insertion of a protective plate. (Below) Postoperative situation with complete closure.

\section{RESULTS}

\section{Part A: Laboratory Experiment}

Single-layered human amniotic membrane exhibited a median tensile strength of $3.46 \mathrm{~N}$ until rupture (mean, $3.563 \mathrm{~N}$; minimum, 1.23 $\mathrm{N}$; maximum, $6.04 \mathrm{~N})$. Double-layered human 
membrane showed a median tensile strength of $8.455 \mathrm{~N}$ (mean, $9.283 \mathrm{~N}$; minimum, $3.05 \mathrm{~N}$; maximum, $16.25 \mathrm{~N}$ ), which was higher than that of single-layered membrane $(p<0.001)$. Four-layer membrane exhibited a median tensile strength of $18.9 \mathrm{~N}$ (mean, $18.825 \mathrm{~N}$; minimum, $11.29 \mathrm{~N}$; maximum, $22.66 \mathrm{~N}$ ), which was higher than that of double-layered membrane $(p<0.001)$.

Single-layered porcine amniotic membrane exhibited a median tensile strength of $1.745 \mathrm{~N}$ until rupture (mean, $1.776 \mathrm{~N}$; minimum, $0.8 \mathrm{~N}$; maximum, $2.97 \mathrm{~N}$ ). Double-layered porcine membrane showed a median tensile strength of 3.82 $\mathrm{N}$ (mean, $4.566 \mathrm{~N}$; minimum, $1.52 \mathrm{~N}$; maximum, $9.41 \mathrm{~N}$ ), which was higher than that of single-layered membrane $(p<0.001)$. Four-layered membrane exhibited a median tensile strength of 9.94 $\mathrm{N}$ (mean, $10.527 \mathrm{~N}$; minimum, $6.04 \mathrm{~N}$; maximum, $17.03 \mathrm{~N})$, which was significantly higher than that of double-layered membrane $(p<0.001)$.

Forces needed for the rupture of human amniotic membrane were higher than those needed for porcine amniotic membrane in each one-, two-, and four-layer technique $(p<0.001)$.

\section{Part B: Animal Experiment}

\section{Clinical Course}

All animals survived with no signs of illness. All oronasal fistulas showed marginal re-epithelialization and no tendency for spontaneous closure at 2 weeks after their creation.

All oronasal fistulas treated with amniotic membrane allograft and autofetal amniotic membrane showed similar wound healing. On day 7 , amniotic membrane was no longer recognizable because of the overgrowth of granulating tissue and fibrin covering. Three of six fistulas in the allograft group and five in the autofetal group were completely closed on day 10. In both groups, all fistulas were completely closed and showed no signs of inflammation on day 76 (Fig. 7). All animals in both groups showed moderate local redness on day 3; this increased in three cases in each group on day 7. A single animal showed moderate local redness on day 10, and no inflammation was observed in any animal in either group on day 76 (Fig. 8). Thus, no difference in clinical course was observable between the animals treated with amniotic membrane allograft or autofetal amniotic membrane.

No small intestinal submucosa was recognizable in animals treated with this material on the seventh postoperative day. One fistula was completely closed on day 10 , and five of the six animals showed a completely closed oral epithelium on day 76 . In the remaining case, a persistent fistula was found with a probe. Local redness was comparable with that of the amniotic membrane groups. Pronounced redness was seen in four animals on day 3 and one animal on day 7 (five with moderate inflammation). Four animals exhibited moderate redness on day 10 , and no signs of inflammation were observed on day 76 .

\section{Histologic Analysis}

Histologic analysis confirmed the clinical observation of completely closed oral epithelium in all samples from both the allograft and the autofetal amniotic membrane groups. Two animals treated with allograft exhibited unilateral fistula tract remnants, and two exhibited bilateral remnants. Three cases of unilateral remnants were found in the autofetal group (Fig. 9), each with histologic signs of mild chronic inflammation on the nasal side. All fistula tract remnants were covered with respiratory epithelium.

All of the animals treated with small intestinal submucosa showed at least unilateral nasal fistula tract remnants. In four cases, remnants were found bilaterally, and in one case, the oral epithelium was not closed, indicating a persisting oronasal fistula. In all but one animal, signs of mild chronic inflammation were evident, and fibrosis occurred in four of these five cases. Table 1 provides an overview of the histological results and details regarding signs of inflammation and fibrosis.

\section{Part C: Clinical Trial}

All patients showed complete closure of the oral epithelium with no signs of inflammation during the follow-up period (Fig. 6, below).

\section{DISCUSSION}

Surgical closure of a cleft palate is intended to establish an intact separation between the oral and nasal cavities. Because of frequent limitations in the amount of local tissues and scarring after previous surgical procedures, the formation of an oronasal fistula is a common complication. ${ }^{27}$ Prognosis for successful closure declines with further attempts at surgical correction. ${ }^{28} \mathrm{~A}$ multitude of closure techniques have been suggested, such as mucoperiosteal flaps, ${ }^{29}$ pedicled tongue flaps, ${ }^{30}$ and free flaps, ${ }^{20}$ but many methods can cause donor-site morbidity. ${ }^{21,31}$

The use of an external biomaterial might represent one way to preserve healthy tissue without the limitations in local quantity and restrictions caused by cicatrization. Donor-site morbidity can be avoided by the use of alternative biological 

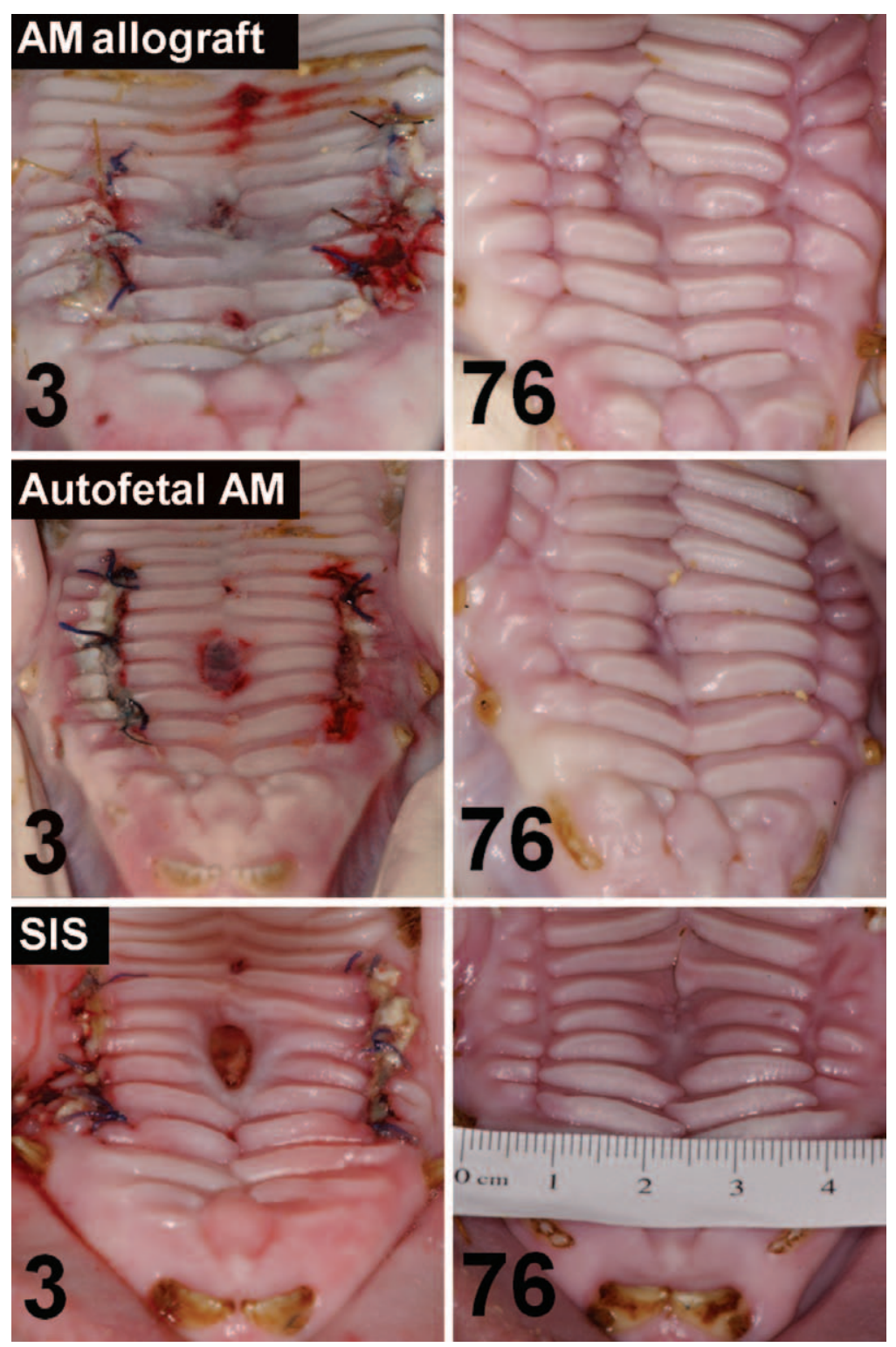

Fig. 7. Postoperative course after palatoplasty in the palate of piglets. Results on postoperative days 3 (left column) and 76 (right column). (Above) Amniotic membrane $(A M)$ allograft used as the reconstruction material. Complete closure of oral epithelium of the iatrogenic oronasal fistula was observed in all animals by day 76. (Center) The same results were observed in the animals treated with autofetal amniotic membrane. (Below) Small intestinal submucosa $(S / S)$ as the transplanted material showed a persistent oronasal fistula in one of the animals.

materials. Biomaterials have become popular in head and neck reconstructive surgery, but only a few publications report their use in oronasal fistula closure.

Acellular dermal matrices were introduced for the closure of palatal clefts in $2003 .{ }^{32}$ Kirschner et al. ${ }^{21}$ described oronasal fistula repair with acellular dermal matrices. In a swine model in the first part of the study and in a clinical trial with nine patients in the second part, the authors achieved convincing results. Complete fistula closure was observed in all patients, despite two patients initially presenting with a dehiscence. Aldekhayel et al. ${ }^{33}$ investigated the available evidence for the usage of acellular dermal matrix in cleft palate repair. The authors concluded that the use of acellular dermal matrices may reduce fistula formation and fistula persistence in palate surgery, 


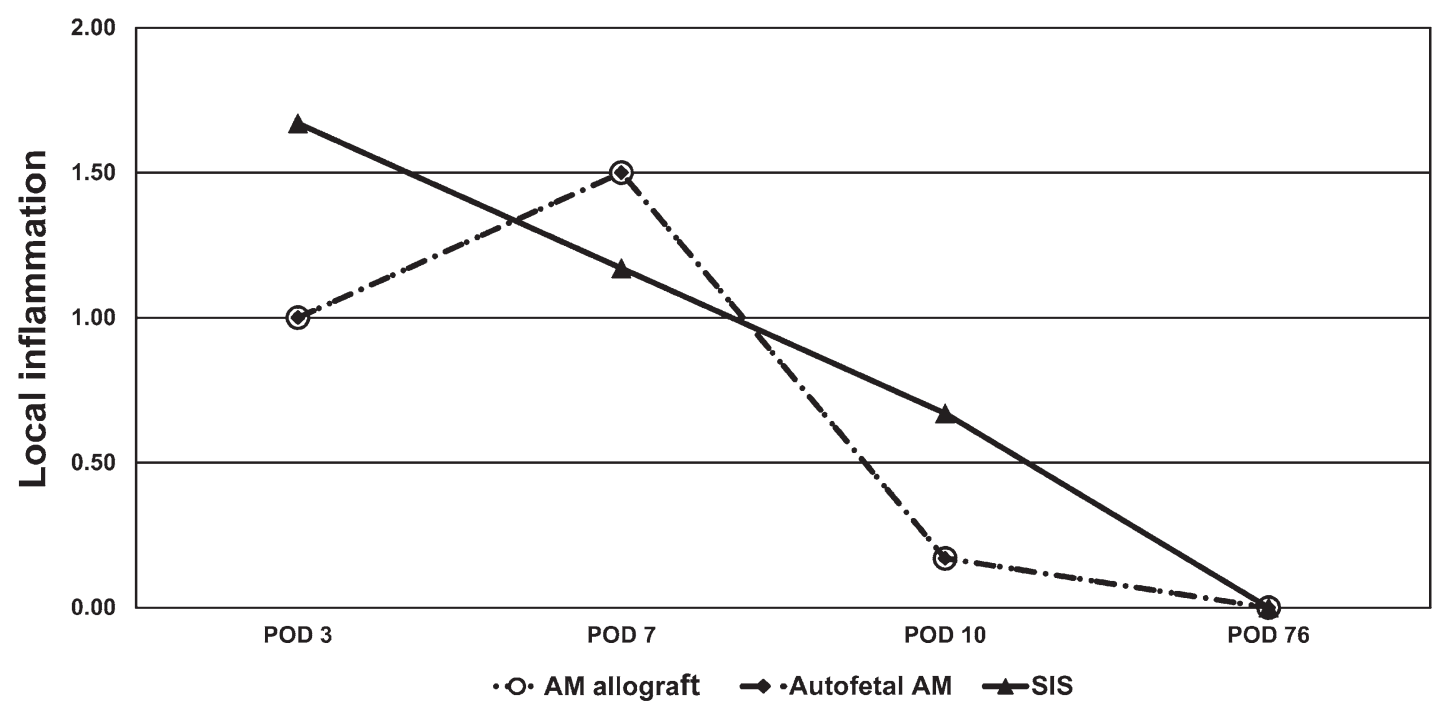

Fig. 8. Chart visualizing the mean values of the clinically observed signs of inflammation ( 0 , none; 1 , moderate; 2, pronounced) after palatoplasty with amniotic membrane (AM) allograft, autofetal amniotic membrane, or small intestinal submucosa (SIS). The axis of the ordinates shows the mean degree of local redness in all animals of a group. The axis of the abscissas displays postoperative days $(P O D) 3,7,10$, and 76 . Although no statistical analysis was carried out because of the small sample size, the diagram indicates that no clinically relevant difference was present in the rate of infections among the groups with amniotic membrane allograft, autofetal amniotic membrane (which showed exactly the same postoperative course with respect to local redness), and small intestinal submucosa, although the local signs of inflammation were slightly higher in the small intestinal submucosa group on postoperative days 3 and 10 .

but they pointed out that prospective, randomized studies are necessary.

Ophof et al. investigated other biomaterials for the reconstruction of palatal defects in dogs, such as tissue-engineered mucosal substitutes containing oral keratinocytes ${ }^{34}$ or scaffolds of cross-linked bovine tendon collagen and glycosaminoglycans. ${ }^{35}$ Transplant loss and cell degradation were observed in the former study.

Amniotic membrane is currently used for reconstruction of the ocular surface, ${ }^{36}$ abdominal wall, ${ }^{26}$ urinary bladder, ${ }^{37}$ leg ulcers, ${ }^{38}$ and burns. ${ }^{39}$ It exhibits anti-inflammatory effects and has low immunogenicity. ${ }^{40}$ This study reports the first

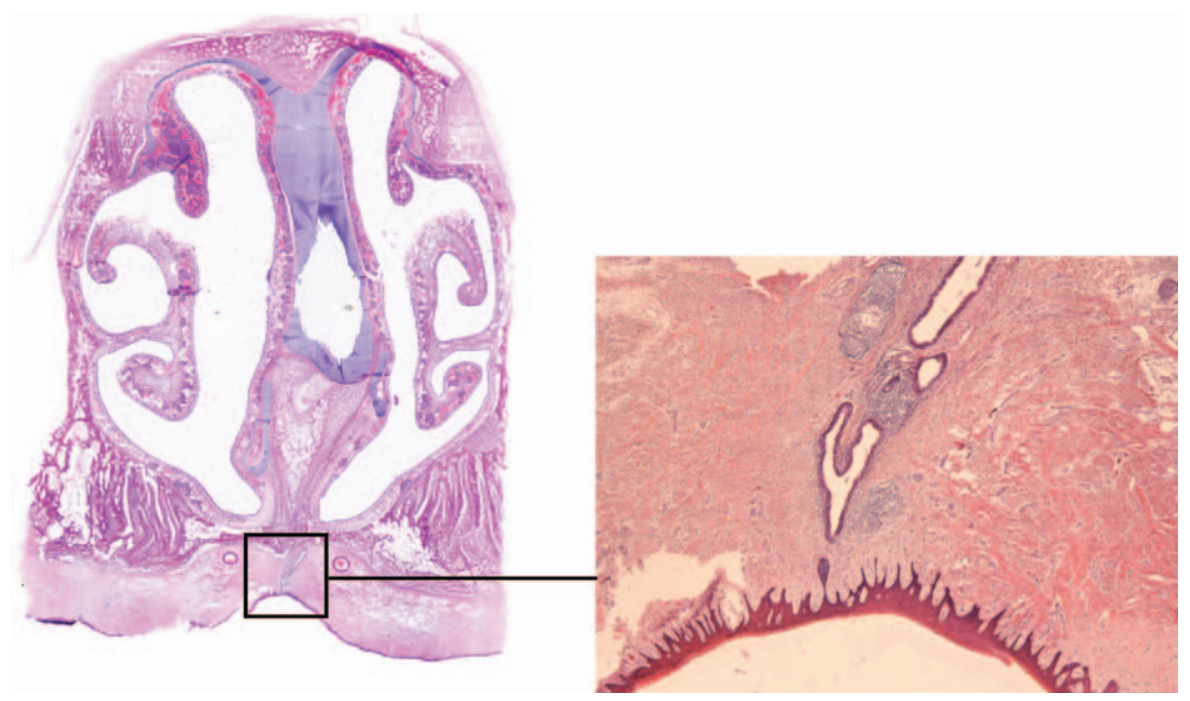

Fig. 9. Fistula tract residue (indicated by box) with mild chronic inflammation after oronasal fistula closure with autofetal amniotic membrane. Transverse section through former palatine defect (hematoxylin and eosin stain). 
Table 1. Results of Histologic Analysis of Palates on Day 76 after Oronasal Fistula Repair

\begin{tabular}{|c|c|c|c|c|c|}
\hline Animal No. & Oral Defect & Nasal Defect Left Side & Nasal Defect Right Side & Inflammation & Fibrosis \\
\hline \multicolumn{6}{|c|}{ Oral fistulas repaired with amniotic membrane allograft } \\
\hline 1 & - & - & - & - & + \\
\hline 2 & _ & - & - & _- & _ \\
\hline 3 & - & + & - & + & - \\
\hline 4 & - & + & +++ & - & + \\
\hline 5 & - & + & + & + & + \\
\hline 6 & - & - & +++ & + & + \\
\hline \multicolumn{6}{|c|}{ Oral fistulas repaired with autofetal amniotic membrane } \\
\hline 7 & - & +++ & - & + & + \\
\hline 8 & - & +++ & _ & + & + \\
\hline 9 & - & - & - & - & - \\
\hline 10 & - & + & - & + & - \\
\hline 11 & - & - & - & - & - \\
\hline 12 & - & - & - & - & + \\
\hline \multicolumn{6}{|c|}{ Oral fistulas repaired with small intestinal submucosa } \\
\hline 13 & - & - & + & - & - \\
\hline 14 & + & ++ & +++ & - & - \\
\hline 15 & - & +++ & + & - & + \\
\hline 16 & - & + & ++ & + & + \\
\hline 17 & - & - & +++ & + & + \\
\hline 18 & - & + & + & + & + \\
\hline
\end{tabular}

Symbols: -, negative; +, positive; ++, pronounced; +++, intensive.

clinical use of amniotic membrane allografts and autofetal amniotic membrane for oronasal fistula repair. Complete fistula closure was observed after transplantation of multilayered autofetal amniotic membrane or amniotic membrane allograft in the animal experiment. No relevant immune reaction or reduced signs of inflammation compared with the group treated with small intestinal mucosa were noted on postoperative days 3 and 10 .

The treatment of patients with amniotic membrane gave excellent results: all fistulas were closed with no signs of inflammation. The results demonstrate that transplantation of autofetal amniotic membrane or amniotic membrane allografts represents an interesting new method for oronasal fistula closure. A potential disadvantage might be the need to fabricate protective plates in order to avoid mechanical damage, but their use and the multilayer technique have solved the previously reported problem of mechanical instability of amniotic membrane. ${ }^{37}$ This study has demonstrated that the stability of an amniotic membrane transplant can be increased by using additional layers.

One interesting opportunity is the storage of autofetal amniotic membrane. Children who are prenatally diagnosed with a cleft palate can later be treated with their own amniotic membrane. Oronasal fistulas are usually not repaired during the infant stage, but cryoconserved amniotic membrane can be stored and later used as an exclusive biomaterial for reconstructive surgery. It can also be used in case of burn wounds or other trauma. The procedures for harvesting and cryoconservation and the clinical use of autofetal amniotic membrane are offered to parents expecting a child diagnosed with a cleft palate.

A drawback of this study is the small number of clinical cases. However, the results of the first patients treated with this method emphasize the encouraging findings from the earlier experimental study arm. A randomized trial with a larger number of cases is necessary to validate these initial results.

\section{CONGILUSIONS}

Amniotic membrane is useful for oronasal fistula repair. It prevents donor-site morbidity and is easy to harvest, economically reasonable, and available in large quantities. The multilayer technique and protective plates solve the problem of mechanical instability. It is now possible to harvest and store the autofetal amniotic membrane of a child with a cleft palate as an exclusive tissue source for later reconstruction. Amniotic membrane allografts can be used without noticeable disadvantages when compared with autofetal amniotic membrane, which is relevant for patients with no reserves of autofetal amniotic membrane.

Marco R. Kesting, M.D., D.D.S., Ph.D. Department of Oral and Maxillofacial Surgery Technische Universität München Ismaninger Str. 22 D-81675 Munich, Germany kesting@mkg.med.tum.de 


\section{ACIKNOWLED GMIENTS}

This work was supported by the German Research Foundation (no. WO 507-1). These data formed part of doctoral theses of Anne Nuber and Leopold Barthel.

\section{REFERENCES}

1. Eberlinc A, Koželj V. Incidence of residual oronasal fistulas: A 20-year experience. Cleft Palate Craniofac J. 2012;49:643-648.

2. Andersson EM, Sandvik L, Semb G, Abyholm F. Palatal fistulas after primary repair of clefts of the secondary palate. Scand J Plast Reconstr Surg Hand Surg. 2008;42:296-299.

3. Majid OW. Persistent oronasal fistula after primary management of facial gunshot injuries. Br J Oral Maxillofac Surg. 2008;46:50-52.

4. Chaudhary SV, Karnik ND, Sabnis GR, Patil MV, Bradoo RA. Extranodal NK/T cell lymphoma presenting as palatal perforation with oronasal fistula. J Assoc Physicians India 2011;59:112-114.

5. Thompson SH, Kola H, Langenegger J, Reyneke J. Oronasal fistula secondary to nasal septal surgery and candidiasis. $J$ Oral Maxillofac Surg. 1985;43:964-966.

6. Nord GA, Rock A, Murphy FJ, Miloslavskiy I, Miller DJ, Wasserman BS. Prosthetic and surgical management of oronasal communications secondary to cocaine abuse. $N Y$ State Dent J. 2012;78:22-25.

7. Phua YS, de Chalain T. Incidence of oronasal fistulae and velopharyngeal insufficiency after cleft palate repair: An audit of 211 children born between 1990 and 2004. Cleft Palate Craniofac J. 2008;45:172-8.

8. Amaratunga NA. Occurrence of oronasal fistulas in operated cleft palate patients. J Oral Maxillofac Surg. 1988;46:834-838.

9. Karling J, Larson O, Henningsson G. Oronasal fistulas in cleft palate patients and their influence on speech. Scand J Plast Reconstr Surg Hand Surg. 1993;27:193-201.

10. van de Wetering A. Repair of an oronasal fistula using a double flap technique. J Vet Dent. 2005;22:243-245.

11. Penna V, Bannasch H, Stark GB. The turbinate flap for oronasal fistula closure. Ann Plast Surg. 2007;59:679-681.

12. Anani RA, Aly AM. Closure of palatal fistula with local double-breasted mucoperiosteal flaps. J Plast Reconstr Aesthet Surg. 2012;65:e237-e240.

13. Garner JM, Wein RO. Use of the palatal flap for closure of an oronasal fistula. Am J Otolaryngol. 2006;27:268-270.

14. Zeidman A, Lockshin A, Berger J, Gold B. Repair of a chronic oronasal defect with an anteriorly based tongue flap: Report of a case. J Oral Maxillofac Surg. 1988;46:412-415.

15. Tunçbilek G, Konaş E, Kayikçioğlu A, Mavili EM. Three-layer oronasal fistula repair with sandwiched mastoid fascia graft. J Craniofac Surg. 2012;23:780-783.

16. Soukup JW, Snyder CJ, Gengler WR. Free auricular cartilage autograft for repair of an oronasal fistula in a dog. $J$ Vet Dent. 2009;26:86-95.

17. Bureau S, Penko M, McFadden L. Speech outcome after closure of oronasal fistulas with bone grafts. J Oral Maxillofac Surg. 2001;59:1408-1413; discussion 1413.

18. Taub PJ, Bradley JP, Kawamoto HK. Closure of an oronasal fistula in an irradiated palate by tissue and bone distraction osteogenesis. J Craniofac Surg. 2001;12:495-499; discussion 500.

19. Christiano JG, Dorafshar AH, Rodriguez ED, Redett RJ. Repair of recurrent cleft palate with free vastus lateralis muscle flap. Cleft Palate Craniofac J. 2012;49:245-248.
20. Chen HC, Ganos DL, Coessens BC, Kyutoku S, Noordhoff MS. Free forearm flap for closure of difficult oronasal fistulas in cleft palate patients. Plast Reconstr Surg. 1992;90:757-762.

21. Kirschner RE, Cabiling DS, Slemp AE, Siddiqi F, LaRossa DD, Losee JE. Repair of oronasal fistulae with acellular dermal matrices. Plast Reconstr Surg. 2006;118:1431-1440.

22. Davis J. Skin transplantation with a review of 550 cases at the Johns Hopkins Hospital. Johns Hopkins Hospital Report 1910;15:310.

23. Walker AB, Cooney DR, Allen JE. Use of fresh amnion as a burn dressing. J Pediatr Surg. 1977;12:391-395.

24. Lee SH, Tseng SC. Amniotic membrane transplantation for persistent epithelial defects with ulceration. Am J Ophthalmol. 1997;123:303-312.

25. Kesting MR, Loeffelbein DJ, Classen M, et al. Repair of oronasal fistulas with human amniotic membrane in minipigs. Br J Oral Maxillofac Surg. 2010;48:131-135.

26. Kesting MR, Loeffelbein DJ, Steinstraesser L, et al. Cryopreserved human amniotic membrane for soft tissue repair in rats. Ann Plast Surg. 2008;60:684-691.

27. Amaratunga NA. Occurrence of oronasal fistulas in operated cleft palate patients. J Oral Maxillofac Surg. 1988;46:834-838.

28. Cole P, Horn TW, Thaller S. The use of decellularized dermal grafting (AlloDerm) in persistent oro-nasal fistulas after tertiary cleft palate repair. J Craniofac Surg. 2006;17:636-641.

29. Honnebier MB, Johnson DS, Parsa AA, Dorian A, Parsa FD. Closure of palatal fistula with a local mucoperiosteal flap lined with buccal mucosal graft. Cleft Palate Craniofac J. 2000;37:127-129.

30. Posnick JC, Getz SB Jr. Surgical closure of end-stage palatal fistulas using anteriorly-based dorsal tongue flaps. J Oral Maxillofac Surg. 1987;45:907-912.

31. Richardson D, Fisher SE, Vaughan ED, Brown JS. Radial forearm flap donor-site complications and morbidity: A prospective study. Plast Reconstr Surg. 1997;99:109-115.

32. Clark JM, Saffold SH, Israel JM. Decellularized dermal grafting in cleft palate repair. Arch Facial Plast Surg. 2003;5:40-44; discussion 45 .

33. Aldekhayel SA, Sinno H, Gilardino MS. Acellular dermal matrix in cleft palate repair: An evidence-based review. Plast Reconstr Surg. 2012;130:177-182.

34. Ophof R, Maltha JC, Kuijpers-Jagtman AM, Von den Hoff JW. Implantation of tissue-engineered mucosal substitutes in the dog palate. Eur J Orthod. 2008;30:1-9.

35. Ophof R, Maltha JC, Kuijpers-Jagtman AM, Von Den Hoff JW. Evaluation of a collagen-glycosaminoglycan dermal substitute in the dog palate. Tissue Eng. 2007;13:2689-2698.

36. Gomes JA, Romano A, Santos MS, Dua HS. Amniotic membrane use in ophthalmology. Curr Opin Ophthalmol. 2005;16:233-240.

37. Iijima K, Igawa Y, Imamura T, et al. Transplantation of preserved human amniotic membrane for bladder augmentation in rats. Tissue Eng. 2007;13:513-524.

38. Mermet I, Pottier N, Sainthillier JM, et al. Use of amniotic membrane transplantation in the treatment of venous leg ulcers. Wound Repair Regen. 2007;15:459-464.

39. Kesting MR, Wolff KD, Hohlweg-Majert B, Steinstraesser L. The role of allogenic amniotic membrane in burn treatment. J Burn Care Res. 2008;29:907-916.

40. Niknejad H, Peirovi H, Jorjani M, Ahmadiani A, Ghanavi J, Seifalian AM. Properties of the amniotic membrane for potential use in tissue engineering. Eur Cell Mater. 2008;15:88-99. 